\title{
EXPERIENCIA DE TRABAJO COLABORATIVO: FORMACIÓN DEL PERSONAL DOCENTE UNIVERSITARIO PARA LA DIVERSIDAD
}

\section{EDUCATIONAL NEEDS IN HIGHER EDUCATION: A COLLABORATIVE WORK EXPERIENCE}

\author{
Martha Gross Martínez* \\ Laura Stiller González **
}

\begin{abstract}
RESUMEN
El presente artículo expone el esfuerzo del trabajo colaborativo entre diferentes instancias de la Universidad de Costa Rica, para lograr articular acciones, tanto a nivel teórico como metodológico, en la formación sobre accesibilidad del personal docente. Se implementó la modalidad de taller, el cual permitió un espacio para compartir experiencias docentes, brindar información y concientizar sobre las necesidades educativas del alumnado, encaminadas al fortalecimiento de una universidad inclusiva, en donde prime el derecho y respeto a la diversidad.
\end{abstract}

PALABRAS CLAVE: FORMACIÓN DE DOCENTES * ENSEÑANZA SUPERIOR * COLABORACIÓN * TRABAJO EN EQUIPO * NECESIDADES EDUCATIVAS * UNIVERSIDAD INCLUSIVA

\section{ABSTRACT}

This article demonstrates the joint effort of different institutions in the University of Costa Rica. In this experience, in which was implemented a workshop modality, the actions from the universitary institution can be integrated with training the professors, both in a theoretical and a methodological level, with the contribution of assertive strategies, congruent with the educational needs of the student body, that look to strengthen an inclusive university where the right and respect towards diversity has great importance.

KEYWORDS: TEACHING TRAINING * HIGHER EDUCATION $*$ PARTNERSHIP $*$ TEAM WORK * EDUCATIONAL NEEDS * INCLUSIVE UNIVERSITY

\footnotetext{
* Escuela de Orientación y Educación Especial de la Facultad de Educación de la Universidad de Costa Rica (UCR). martha.gross@ucr.ac.cr

** Centro de Asesoría y Servicios al Estudiante con Discapacidad (CASED) de la Universidad de Costa Rica (UCR). laura.stiller@ucr.ac.cr
} 


\section{INTRODUCCIÓN}

Las transformaciones conceptuales sobre accesibilidad se gestan de forma coyuntural, a través de los diferentes procesos y posiciones en pro de la igualdad de oportunidades. En este contexto, la Universidad de Costa Rica ha tenido un papel protagónico por generar propuestas de cambio sobre las demandas de las y los estudiantes con discapacidad, aun antes de la promulgación de la Ley 7600 de Igualdad de Oportunidades para las personas con discapacidad en Costa Rica.

Posteriormente, en el marco internacional, la Convención sobre los Derechos de las Personas con Discapacidad, en su Protocolo facultativo, artículo 9, define accesibilidad como:

(...) medidas pertinentes para asegurar el acceso de las personas con discapacidad, en igualdad de condiciones con las demás, al entorno físico, el transporte, la información y las comunicaciones... Estas medidas que incluirán la identificación y eliminación de obstáculos y barreras de acceso... (onu, 2007: 17).

Con el fin de que este proceso se realice en forma efectiva, la Convención, en su artículo 24 sobre educación, señala que el Estado debe asegurar que se brinden medidas de apoyo personalizadas en un ambiente educativo que propicie la inclusión de la población estudiantil con discapacidad (onu, 2007).

En este sentido, la Escuela de Orientación y Educación Especial, en concordancia con su propuesta (2012), genera conocimiento e innovación en la formación de profesionales que contribuyan con la comprensión de la realidad socio-educativa y la promoción de acciones favorecedoras del desarrollo de la persona en su contexto. Como parte de este compromiso ante la comunidad universitaria, apoya $y$ fortalece la docencia, con acciones que propician la igualdad y equiparación de oportunidades en el contexto de la Universidad de Costa Rica.

Por su parte, el Centro de Asesoría y Servicios a Estudiantes con Discapacidad (cased), unidad operativa de la Oficina de Orientación, asume el reto en la toma de decisiones y acciones sobre el proceso de inclusión educativa de la población estudiantil con necesidades educativas asociadas o no, a la discapacidad. Al respecto, asesora a la comunidad universitaria en la temática de accesibilidad $y$ brinda a esta población estudiantil, los servicios $y$ apoyos requeridos, tanto en el proceso de admisión como en la permanencia. Así, el CASED se consolida como una instancia que apoya, promueve y coordina acciones para la participación plena en igualdad de oportunidades del estudiantado con discapacidad.

Este compromiso de la Universidad en procura de la equidad, ha permitido que las instancias señaladas, conjuntamente con el Departamento de Didáctica Universitaria (Dedun), Centro de Evaluación Académica (cEa) y la Maestría Centroamericana en Estudios Interdisciplinarios sobre Discapacidad, articulen sus esfuerzos con una meta en común: la capacitación del personal docente en materia de accesibilidad. A partir de esta vinculación, se realiza una propuesta de taller a través del cual se posibilita un espacio de participación, comunicación e intercambio de conocimientos y estrategias transformadoras para la práctica docente.

Ante el compromiso señalado, se desarrolla, a partir de 2007, el Taller de Necesidades Educativas Especiales, el cual pretende visualizar el trabajo colaborativo como un sustento dinámico para la consecución de nuevos retos en materia de accesibilidad $y$ discapacidad.

\section{ANTECEDENTES}

En el marco de su Estatuto Orgánico, la Universidad de Costa Rica ha realizado una serie de esfuerzos en la construcción de una universidad accesible al plantear:

El propósito de la Universidad de Costa Rica es obtener las transformaciones que la sociedad necesita para el logro del bien común, mediante una política dirigida a la consecución de una verdadera justicia social, del desarrollo integral, de la libertad plena $y$ de la total independencia de nuestro pueblo (Consejo Universitario, 1974, artículo 3). 
Para cumplir con este propósito, la Universidad ha definido políticas institucionales que responden a la temática en materia de equidad $y$ accesibilidad, las cuales favorecen la equiparación de oportunidades y permiten la participación de la población universitaria en igualdad de condiciones. Por su parte, en los Principios y Políticas Institucionales del 2009, se indica de forma contundente, que la Universidad impulsará acciones dirigidas a reducir y eliminar cualquier tipo de desigualdad en la comunidad universitaria, con el fin de que contribuyan a consolidar una cultura de justicia, equidad $y$ de respeto a las personas (Consejo Universitario, 2008).

En este proceso de igualdad de oportunidades se han generado acciones institucionales vinculadas a la accesibilidad, llevadas a cabo por:

$\diamond \quad$ CASED, brindando apoyo y servicios a la población con necesidades educativas $y$ capacitación a la comunidad universitaria.

$\diamond \quad$ La Escuela de Orientación y Educación Especial, en la formación de profesionales y propiciando la igualdad de oportunidades.

$\diamond \quad$ El Sistema de Bibliotecas, Documentación e Información (sibdi), con el Programa de Bibliotecas Accesibles.

$\diamond \quad$ La Maestría Centroamericana en Estudios Interdisciplinarios sobre Discapacidad, en la formación de profesionales.

$\diamond \quad \mathrm{El}$ cEA, con sus aportes en la consecución de su misión de contribuir al mejoramiento académico y las acciones cotidianas.

$\diamond \quad$ El dedun, en la formación de docentes universitarios y universitarias.

Este trabajo interdisciplinario de las diferentes instancias universitarias evidencia el interés institucional y el aprovechamiento de recursos, ya que cada una de ellas participa desde una perspectiva particular, como lo manifiesta D`Antoni:

Parece positivo de un lado que exista interés institucional acerca de temas ligados a la inclusión educativa, mientras que por el otro lado se espera que no haya duplicación de funciones, más bien construcción de redes multiplicadoras de los esfuerzos producidos (2008: 6).

Para articular estos esfuerzos institucionales, atendiendo lo dispuesto en la Ley 7600 de Igualdad de Oportunidades para las Personas con Discapacidad en Costa Rica, el Consejo Universitario acuerda lo siguiente:

Pedir al Departamento de Docencia Universitaria de la Escuela de Formación Docente de la Universidad de Costa Rica que en el programa del curso de Didáctica Universitaria se incluyan contenidos relacionados con la aplicación de lo dispuesto en la Ley 7600 de Igualdad de Oportunidades para las Personas con Discapacidad en Costa Rica. Además, solicitar la colaboración de este Departamento, a fin de que se organicen actividades de capacitación en coordinación con la Maestría Centroamericana en Estudios Interdisciplinarios sobre Discapacidad y el Centro de Asesoría y Servicios a Estudiantes con Discapacidad de la Oficina de Orientación de la Vicerrectoría de Vida Estudiantil, con el objetivo de actualizar al profesorado en servicio $y$ en formación sobre el tema de la flexibilización del currículo universitario en aras de atender esta población (Consejo Universitario, 2004, Sesión ordinaria nro. 4919, punto 8).

En atención a este acuerdo del Consejo Universitario, el Dedun y la Maestría Centroamericana en Estudios Interdisciplinarios sobre Discapacidad plantearon un taller para formación docente en la temática de accesibilidad al espacio físico, a la información y al proceso educativo. Es así como, a partir del segundo semestre de 2007, se acuerda formar una comisión de personas expertas para revisar y elaborar dicha propuesta. La comisión se conformó por representantes de las siguientes instancias:

$\diamond \quad$ Maestría Centroamericana en Estudios Interdisciplinarios sobre Discapacidad 
$\diamond \quad$ Centro de Asesoría y Servicios a Estudiantes con Discapacidad (CASED)

$\diamond \quad$ Escuela de Orientación y Educación Especial

$\diamond \quad$ Centro de Evaluación Académica (сЕA)

$\diamond \quad$ Departamento de Didáctica Universitaria (DEDUN)

Las labores de las instancias involucradas en la construcción del taller, cada una desde su competencia y experticia, tuvo como finalidad común, el respeto a la diversidad en la dinámica universitaria. Por lo tanto, el taller de necesidades educativas especiales se constituye en un espacio para implementar la articulación de entidades institucionales en pro de la formación del personal docente universitario en la temática de discapacidad $y$ accesibilidad.

\section{COLABORACIÓN Y APRENDIZAJE}

Considerando las características e intención formadora de esta experiencia y sus implicaciones en el quehacer universitario, en la propuesta de trabajo se incluyó la incidencia e interacción de las diferentes instancias universitarias vinculadas con el compromiso de dar respuesta a la diversidad en el quehacer docente. Así, se hizo hincapié en el trabajo conjunto y de colaboración, como bien lo afirman Arnaíz, Herrero, Garrido y De Haro:

El grupo colaborativo, donde todos los componentes participan según sus habilidades en la toma de decisiones, y contribuyen en la medida de sus habilidades e intereses, de manera que todos son responsables de la calidad de lo producido por el grupo (1999: 2).

Desde esta perspectiva, el trabajo colaborativo motivó e indujo al intercambio de conocimientos, acciones y recursos, entre participantes en la construcción del taller. Por lo tanto, este proceso permitió no solo organizar el contexto educativo, sino interpretarlo desde las experiencias y aportes de cada instancia. El trabajo colaborativo dio lugar a un ambiente de aprendizaje, en el cual se dieron retos de planificación, definición de roles y responsabilidades, aspectos que a la vez, facilitaron el proceso de implementación del Taller. Así, de las fortalezas de cada instancia se generó una colaboración continua y comprometida con los objetivos de la docencia en una universidad inclusiva y accesible.

Por otra parte, un aspecto que enriqueció la participación colaborativa de las instancias vinculadas fue la interdisciplinariedad de las mismas, trabajando por un objetivo común. Esto fortaleció la labor realizada y los roles del equipo desde las disciplinas y quehaceres particulares, así como propició el intercambio de información, conocimientos y recursos en la construcción y ejecución del Taller.

Para promover acciones concretas hacia las demandas y necesidades de formación del personal docente, se tomó como insumo el marco teórico y conceptual en materia de discapacidad y accesibilidad. De esta manera, la organización y sustento del Taller respondió a un proceso de colaboración sistemática, en el cual:

...puede inferirse que la atención a la diversidad no se centra únicamente en determinados alumnos con necesidades educativas especiales (n.e.e.) asociadas a déficits, sino que es una tarea institucional que compete a la comunidad educativa, con la finalidad de dar la respuesta más adecuada a los alumnos... (Clark y otros, 1997, 1998; Gartner y Lipsky, 1987; Norwich, 1996, citados por Arnáiz et ál., 1999:1).

Considerando las características del conocimiento que se pretendía abarcar en el Taller-el entorno, la población estudiantil, la intención educativa $y$ las estrategias de aprendizaje-, el trabajo colaborativo permitió dar respuesta a un planteamiento didáctico, el cual se conceptualizó como un modelo de aprendizaje interactivo (Maldonado, 2007) que conjugó esfuerzos institucionales y competencias disciplinarias (psicólogas, educadoras especiales y especialistas en accesibilidad) para lograr construir el Taller.

La fundamentación, estructura y programación del Taller generaron aportes significativos de las experiencias y prácticas de las instancias participantes, con un fuerte 
compromiso hacia la consecución asertiva y dinámica de una propuesta didáctica que fuera coherente con un paradigma innovador que visualizara la diversidad como un elemento prioritario en el quehacer docente.

El trabajo realizado partió del concepto de que la función docente implica asumir un rol de mediador del aprendizaje, entendiendo esto como las diferentes acciones que se llevan a cabo en el aula para que el estudiantado se acerque $y$ comprenda el objeto de estudio. De esta forma, como bien lo afirma Bell y Day, citado por Sánchez (2002), el desarrollo de la docencia universitaria no solo se enfoca desde una perspectiva individual o personal, sino también grupal e institucional. Esta última plantea el análisis de las necesidades, la priorización de estas y la toma de decisiones como respuesta realizada en forma conjunta. Esto se logró mediante la interrelación de las docentes $y$ los docentes participantes en la búsqueda de alternativas de accesibilidad al currículo, según las necesidades educativas del estudiantado.

De esta manera, el Taller alcanzó una puesta en común de criterios a partir de las vivencias de cada experiencia docente, considerando los diferentes enfoques disciplinarios (Odontología, Terapia Física, Educación Física, Bibliotecología, Inglés, Psicología, Zootecnia, entre otras).

En el Taller de Necesidades Educativas Especiales se pretendió orientar el proceso de aprendizaje con la experiencia en el campo de la docencia, con ejemplos y vivencias que pudieran ubicar al grupo de docentes con su práctica. Con esto se entiende que el rol docente universitario en el Taller pasó por diferentes acciones, en cada una de las cuales revisó conceptos y abrió espacio de realimentación, análisis y síntesis del proceso. Esto condujo a la interiorización del conocimiento, evidenciada en la riqueza de aportes del trabajo realizado; por ello se planteó de forma consensuada, que el personal docente se apropiara de la experiencia y accionar dentro de un contexto diverso.

\section{TALLER DE NECESIDADES EDUCATIVAS ESPECIALES}

A partir del 2007, el Taller de Necesidades Educativas Especiales se implementó, con el objetivo de capacitar al profesorado universitario en el tema de la discapacidad y en la atención a estudiantes con discapacidad; además, con el propósito de generar, dentro de la comunidad universitaria, conciencia y acciones encaminadas a reconocer los deberes y derechos de la población estudiantil con necesidades educativas asociadas o no a discapacidad.

Se utilizó la metodología de taller, ya que facilita la integración de la teoría y la realidad de una manera dinámica y participativa.

Entre los objetivos específicos por alcanzar están:

1. Promover dentro de la comunidad universitaria, acciones encaminadas a reconocer las necesidades, deberes $y$ derechos de las personas con discapacidad.

2. Sensibilizar al profesorado universitario con respecto a la realidad de la persona con discapacidad.

3. Propiciar procesos de reconocimiento de los derechos $y$ deberes del estudiantado con discapacidad.

Los aspectos claves que se plantearon para el Taller fueron los siguientes:

$\diamond \quad$ Información sobre discapacidad: se consideró importante que las personas participantes en el Taller contaran con información conceptual $y$ datos sobre discapacidad $y$ accesibilidad.

$\diamond \quad$ Marco legal sobre discapacidad: enmarcó la igualdad de oportunidades de acceso a la educación superior para estudiantes con necesidades educativas asociadas o no a discapacidad, en la legislación nacional e internacional (Constitución Política de la República, en su capítulo de Derecho a la Educación; la Ley 7600 de Igualdad de Oportunidades para las Personas con Discapacidad, 1996, y la Ley 8661 Convención sobre los Derechos de las Personas con Discapacidad, 2008). Además, se consideran la reglamentación particular de la Universidad de Costa Rica, como: el Estatuto Orgánico de la Universidad (1974), las políticas 
institucionales en el campo de la discapacidad aprobadas por el Consejo Universitario de la Universidad de Costa Rica (2010-2014) y el Reglamento de Régimen Académico Estudiantil (2011).

$\diamond \quad$ Sensibilización: a través del Taller se intentó abordar la temática de accesibilidad y discapacidad, no solo como datos que se deben conocer, sino vivenciar esta problemática desde la experiencia, el sentimiento, la identificación y la aceptación de la diversidad.

De esta forma, estos aspectos se plantearon como un proceso activo y transformador, que ofrece alternativas de acercamiento hacia el aprendizaje de estrategias facilitadoras de respuestas a la atención de la diversidad de necesidades de la población estudiantil. Por lo tanto, incluye momentos de conceptualización, vivencias y reflexión de la práctica docente en una universidad inclusiva.

El Taller se estructuró en cuatro módulos, dos presenciales $y$ dos virtuales, con una duración de 4 horas cada uno a lo largo de un mes. Cada módulo se realizó una vez por semana, en el siguiente orden: una sesión presencial, dos virtuales y una sesión presencial para finalizar.

Módulo I: el objetivo fue sensibilizar a los participantes sobre la temática de la discapacidad de una forma dinámica y conceptual. Además, se dio información sobre la metodología de trabajo del Taller.

Módulo II: el objetivo fue brindar información sobre legislación y estado de la cuestión sobre discapacidad en general y específico en la Universidad de Costa Rica, conceptos y antecedentes. Se ofreció por medio de la plataforma virtual con una guía de trabajo.

Módulo III: el objetivo fue brindar información sobre legislación y estado de la cuestión sobre discapacidad en el ámbito de la Universidad de Costa Rica, conceptualización de los diferentes tipos de discapacidad, servicios $y$ acciones en el aula.

Módulo IV: charla sobre los esfuerzos de la Universidad de Costa Rica en incorporar el tema de la discapacidad en los planes de estudio y se brindó un espacio de retroalimentación con los participantes para el cierre del Taller.

Las personas que conformamos esta comisión nos dimos a la tarea de construir un taller, empezando por la revisión, recopilación de información y el establecimiento de los pilares que sustentaron desde el inicio al Taller de Necesidades Educativas Especiales. Al respecto, se procedió a la obtención de información y normativa en la temática de discapacidad y la búsqueda de medios para favorecer la sensibilización de reconocer una realidad distinta a la propia, un acercamiento a la vivencia del otro que previene conductas discriminatorias como exclusión y violencia social (Montoya, 2007).

\section{ESTRATEGIAS IMPLEMENTADAS EN EL TALLER}

A partir de los aspectos señalados del Taller, se generaron una serie de propuestas metodológicas con el fin de integrar el conocimiento, las perspectivas, los enfoques y los principios sobre las temáticas por desarrollar. Cada uno de estos conceptos se vinculó el uno con el otro, se realizó un continuum con los principios y políticas que los sustentan. Con la interrelación y vinculación de estos, se favoreció un proceso de enseñanza-aprendizaje fluido ante los requerimientos propios de las estrategias realizadas, enriqueciendo y fortaleciendo la formación de las y los participantes.

Así, la experiencia del proceso de construcción del Taller permitió desarrollar una serie de estrategias que facilitaron un intercambio dinámico entre la información brindada $y$ las vivencias del personal docente en su quehacer en las aulas. De esta forma, las estrategias se conceptualizaron como: “... el proceso en que se sintetiza tanto los componentes de reflexión y de acción y en ella, ambos procesos se encuentran y se retroalimentan" (Cascante y Francis, 2011: 12).

A partir de esta conceptualización, se desarrolló un trabajo en el cual el planteamiento de las estrategias implicó desafíos para descubrir y encontrar alternativas diferentes, en la diversidad de los espacios didácticos de 
las aulas universitarias. Con la aplicación de estas y la participación docente, se logró la construcción de propuestas de mediación en el aula, a nivel individual y grupal. Este proceso los llevó a plantear soluciones o profundizar en aquellos aspectos relacionados con una práctica docente inclusiva, con la cual se reconoce, a partir de las necesidades educativas, la realidad del estudiantado y se responde con la aplicación de estrategias de acceso al currículo para su desarrollo. De esta manera, la dinámica de las estrategias implementadas durante el Taller, permitió la operacionalización de los conocimientos y técnicas adquiridas, en la búsqueda del desarrollo del profesorado, la experiencia y acción docente, además de la realimentación teórica y práctica del proceso de aprendizaje en el aula universitaria.

Por otro lado, la selección de las estrategias aplicadas en el Taller, implicó un análisis detallado de los siguientes aspectos:

\section{Objetivos del taller}

2. Material didáctico y recursos audiovisuales disponibles (casos de estudio, artículos, documentos, guías de estudio, juegos, presentaciones digitales $y$ videos), proporcionados por las instancias vinculadas con la construcción del taller

3. Tiempo designado a las actividades preparadas

La articulación de estos aspectos y las estrategias propiciaron la interacción continua entre conocimiento (conceptos, legislación, apoyos y recursos existentes en materia de discapacidad, accesibilidad, necesidades educativas e inclusión educativa), la experiencia del personal docente en su práctica y en las actividades diseñadas para el logro de los objetivos del Taller, generando un proceso de aprendizaje dinámico y asertivo.

A continuación, se presenta una síntesis de las estrategias implementadas:

A) Sesiones bimodales: esta estrategia consistió en cuatro sesiones de trabajo, dos en forma presencial y dos virtuales. En ellas se abarcaron dinámicas de concientización y se brindó información sobre normativa, conceptos y servicios que ofrece la Universidad para la población de estudiantes con necesidades educativas.

La estructura del Taller inició con una sesión presencial, con el objetivo de sensibilizar a las personas participantes. Se realizaron actividades como el foto mural, "me pongo en los zapatos del otro" $y$ videos, los cuales permitieron un primer acercamiento a la temática a tratar. De esta manera, el grupo de docentes participantes inició el proceso de motivación hacia el dominio y conocimiento de los contenidos del Taller. Posteriormente, se brindó información sobre la legislación y normativa universitaria a través de una charla explicativa, la cual se completó con una dinámica de preguntas que fueron respondidas de forma individual; asimismo, se abarcaron inquietudes, por ejemplo, la perspectiva del docente ante las disposiciones del uso de ayudas técnicas en el aula, tal y como lo dicta la legislación, obligaciones señaladas por la Ley 7600 , para que se cuente un entorno universitario accesible para estudiantes con discapacidad, etc.

En la siguiente sesión, se presentaron los servicios que ofrece la Universidad, acciones en el aula $y$ procesos evaluativos adecuados al estudiantado con necesidades educativas.

Para finalizar, se abrió un espacio de retroalimentación sobre los esfuerzos y acciones que propician el desarrollo de una universidad inclusiva e información sobre la temática de accesibilidad $y$ discapacidad desde la perspectiva de docentes de sus respectivas unidades académicas; esto con el fin de visibilizar los logros alcanzados a nivel institucional y plantear las inquietudes y posibles respuestas a las necesidades evidenciadas en la discusión.

B) Módulos de aprendizaje: esta propuesta metodológica consistió en cuatro módulos de aprendizaje, dos desarrollados en forma bimodal, a través de la Plataforma Virtual de la Universidad de Costa Rica y dos de manera presencial. Con el fin de ofrecer espacios específicos sobre diversos aspectos vinculados con las necesidades educativas y su impacto en el aula universitaria. Cada módulo implementó 
un tema en particular y se designaron de la siguiente manera:

$\diamond \quad$ Módulo 1. Marco legal y reglamentario, historia y conceptos claves

$\diamond \quad$ Módulo 2. Sensibilización, acercamiento. Necesidades educativas especiales

$\diamond \quad$ Módulo 3. Vivencias del personal docente, adecuaciones curriculares. Inclusión

$\diamond \quad$ Módulo 4. Propuestas, evaluación y renovación continua del Taller

Cada módulo se apoyó en una variedad de recursos, actividades y guías de trabajo, como por ejemplo, realizar entrevistas a diferentes instancias universitarias (Programa Regional de Sordera, Sistema de Bibliotecas e Información, Centro de Asesoría y Servicios a Estudiantes, Vicerrectoría de Vida Estudiantil, etc.) sobre servicios de apoyo y recursos; recorrer los edificios de las unidades académicas $y$ entrevistar al director, directora o asistente administrativo, con el fin de que las personas participantes conocieran de manera vivencial sobre la diversidad en el contexto del aula universitaria. De esta manera, estos módulos permitieron identificar las necesidades derivadas de condiciones de discapacidad o de aprendizaje, en contextos variados dentro del campus universitario, a fin de favorecer su conocimiento teórico y práctico al respecto, así como, las posibles alternativas de apoyo a la población con necesidades educativas en el entorno universitario.

Con esta estrategia de aprendizaje, se promovió un espacio de reflexión y análisis crítico de la práctica docente ante el reto de la diversidad, asimismo se fortaleció la conceptualización y la vivencia de una universidad inclusiva.

C) Docencia inclusiva. Desafíos y acciones en la educación universitaria: esta propuesta surgió como una respuesta en la cual “... la Universidad de Costa Rica reconoce la pertinencia de incidir en el cambio social mediante la consecución de una Universidad inclusiva y accesible" (Departamento de Docencia Universitaria, 2011: 1).
En esta trayectoria - que inicia desde el 2007- el Taller de Necesidades Educativas Especiales expandió su visión, con el fin de proyectarse de forma más vivencial $y$ vincularse con las experiencias particulares de la población docente. Así, el Taller se transformó en una propuesta que fortalece la fundamentación de las necesidades educativas; en una universidad donde prevalece la diversidad, brinda un espacio de encuentro para docentes, en el cual puedan reflexionar sobre la diferencia y la singularidad de esta población estudiantil.

\section{RECURSOS Y ACTIVIDADES DE APOYO}

Con el fin de realizar un análisis de los procesos educativos en el contexto universitario, se utilizaron una serie de recursos que promovieron la comprensión y acción sobre la docencia inclusiva. A continuación se describen algunos de ellos:

\section{A) VIDEOS DE EXPERIENCIAS DE PERSONAS CON DISCAPACIDAD}

Para esto se utilizó una producción de videos del Centro de Evaluación Académica (CEA), elaborados con el fin de cumplir con los lineamientos del Consejo Universitario en el marco de "sensibilización sobre la discapacidad en educación", como parte de un proyecto de extensión docente, realizado del 2005 al 2007, con la Vicerrectoría de Acción Social (Código ED 1697):

El propósito de los videos es tener un material de apoyo que sirva como medio a las personas asesoras del CEA en el proceso de sensibilización del personal docente de la comunidad universitaria o de las instancias que así lo requieran. Los videos nos acercan a la comprensión de las personas con alguna discapacidad $y$ al entendimiento de los principios fundamentales que rigen la dignidad propia de los seres humanos sin admitir relativismos de edad, etnia, sexo o discapacidad (Calderón, 2007: 1). 


\section{B) ESTUDIO DE CASOS}

Se utilizó esta técnica como un medio para profundizar sobre las situaciones de la población estudiantil con necesidades educativas en el contexto universitario. Se presentaron varios casos anónimos extraídos de las experiencias reales de estudiantes de la Universidad de Costa Rica. Se tomaron en cuenta las diferentes condiciones de aprendizaje asociadas o no a discapacidad - por ejemplo, situaciones de estudiantes con discapacidad visual, auditiva, motora, condición de déficit atencional, emocional o aprendizaje-, lo cual permitió un acercamiento hacia la diversidad en el entorno universitario. Con la propuesta de los casos, se plantearon preguntas dirigidas al análisis y la búsqueda de respuestas asertivas hacia el acceso al currículo.

\section{C) FORO}

Se organizó un encuentro abierto a la comunidad universitaria con especialistas en el tema de los derechos de las personas con discapacidad, con el fin de abrir un espacio de intercambio y discusión. Se generó una reflexión sobre los alcances en materia de legislación a nivel nacional e internacional, así como, la participación de las personas con discapacidad, el impacto en las acciones y normativas universitarias para el fortalecimiento de una universidad accesible. Los invitados e invitadas al foro expusieron sobre la Convención de los Derechos de las Personas con Discapacidad, enfocándola al ámbito educativo y los participantes aclararon sus dudas al respecto.

\section{D) CONVERSATORIO}

Esta técnica se utilizó con el propósito de compartir experiencias e información sobre accesibilidad en la Universidad. Se contó con la participación de estudiantes con necesidades educativas asociadas o no a la discapacidad, quienes expusieron y relataron sus vivencias, contextualizándolas dentro del aula, así como, las oportunidades $y$ limitaciones con las que se han encontrado en las diferentes situaciones de aprendizaje.
El conversatorio permitió el intercambio entre el estudiantado y participantes del Taller, ya que se construyó un diálogo ágil hacia el conocimiento $y$ oportunidades de accesibilidad en el entorno universitario.

\section{E) JUEGO DE MESA “LAS TAREAS DE LA JUNGLA"}

"Este juego se propone presentar situaciones paradójicas, donde el ámbito sugerido es el de una escuela primaria de diferentes animales de la selva, a los que el maestro les asigna tareas de manera indiferenciada" (D’Antoni y Vargas, 2011: 31). Al considerar las condiciones particulares del y la estudiante, buscando creativamente las adecuaciones requeridas, permitió la reflexión de las y los participantes del Taller con respecto a las demandas curriculares.

\section{VALORACIÓN DE LA EXPERIENCIA}

La docencia universitaria se transforma de manera constante, por lo que la formación y actualización es fundamental. En este sentido y con la finalidad de cumplir con el lineamiento del Consejo Universitario, de formar a docentes en la temática de accesibilidad, se implementó el Taller de Necesidades Educativas Especiales.

Este taller es muestra del trabajo colaborativo que se obtuvo a través de la participación y cooperación de las siguientes instancias universitarias: DEDUN, CEA, CASED, la Maestría Centroamericana en Estudios Interdisciplinarios sobre Discapacidad y la Escuela de Orientación y Educación Especial. El propósito de esta estrategia fue fortalecer las acciones docentes, en procura de la igualdad de oportunidades y el reconocimiento de la diversidad como un elemento enriquecedor en el aula.

A partir de este esfuerzo conjunto, el Taller propició espacios de información, discusión, reflexión y concientización, como lo manifiestan sus participantes en las evaluaciones del Taller:
- Salgo del curso como un ser más consciente y sensible, y con más deseo de ser parte de las soluciones.
-En relación con lo que me dejó el curso, considero que me sirvió de 
plataforma para darme cuenta de 10 que nos falta en muchos aspectos por mejorar dentro de nuestra escuela en relación a la inclusividad.

De igual manera, las estrategias y recursos de apoyo utilizados (dinámicas, videos, foto mural, "me pongo en los zapatos del otro", las tareas de la jungla, entre otros), permitieron la reflexión sobre una docencia crítica y respetuosa de las necesidades educativas de la población estudiantil. La aplicación de las estrategias mediadoras de aprendizaje permitió el contacto entre la realidad de la accesibilidad $y$ las necesidades educativas en el entorno universitario, así como, la discusión conceptual de diferentes situaciones $y$ prácticas docentes.

Además, esto propició la divulgación de información sobre los servicios y apoyos que ofrece la Universidad a la población estudiantil con necesidades educativas, como son el Programa de Bibliotecas Accesibles y el CASED, lo cual - a su vez - constituye un recurso valioso para el ejercicio de la docencia universitaria:

- Me enteré de los recursos con que cuenta la Universidad de Costa Rica para atender de una manera más apropiada a las personas con alguna discapacidad física y estos recursos están disponibles para toda la comunidad universitaria.

Esta experiencia brindó la oportunidad, al grupo docente, de acercarse a su unidad académica e indagar sobre su accesibilidad, proponer cambios o apoyar acciones que transformen la accesibilidad en esta. Es así como las personas participantes de algunas escuelas plantearon utilizar la información del Taller para procesos de acreditación, promover acciones como el Proyecto de Museos Accesibles y capacitar al resto del personal docente, como lo indica uno de los participantes en el taller: "-es importante que este curso lo lleven todos los de la Universidad".

La propuesta colaborativa a través de la modalidad de taller permitió el intercambio y la exploración de alternativas y respuestas para mediar la acción docente desde lo conceptual y teórico, hasta lo dinámico, reflexivo y vivencial. Se posibilitó el respeto a la diversidad docente $y$ estudiantil, propiciando enlaces entre instancias, servicios y acciones para favorecer la equiparación de oportunidades en el entorno universitario.

El trabajo colaborativo facilitó la vinculación entre distintas instancias universitarias, con un enfoque interdisciplinario, lo cual propició la optimización de recursos en beneficio de la población estudiantil universitaria con necesidades educativas.

Apoyar acciones de este tipo, refuerza la calidad de la docencia universitaria, ya que proporciona mayores estrategias a sus participantes, siendo el dinamismo y creatividad de esta propuesta un proceso más hacia el logro de una universidad inclusiva que responde a la diversidad estudiantil. El Taller de Necesidades Educativas Especiales se ha constituido en una herramienta de formación docente en materia de accesibilidad $y$ un ejemplo de que el trabajo colaborativo institucional es posible.

\section{REFERENCIAS}

Arnáiz, P.; Herrero, A. J.; Garrido, C. y De Haro, R. "Trabajo colaborativo entre profesores y atención a la diversidad". Comunidad Educativa 263-264. 1999: 29-35.

Calderón, L. Guía de trabajo para videos. Costa Rica: Centro Evaluación AcadémicaUniversidad de Costa Rica, 2007.

Cascante, N. y Francis, S. "Capítulo 1. Caminos hacia la innovación docente: las estrategias didácticas del profesorado universitario". Nuevos formatos para la función docente universitaria. San José, Costa Rica: Departamento de Docencia Universitaria-Escuela de Formación Docente-Universidad de Costa Rica, 2011.

Consejo Universitario. Estatuto Orgánico de la Universidad de Costa Rica. Universidad de Costa Rica, 1974.

Consejo Universitario. "Punto 8". Sesión ordinaria nro. 4919. 2004.

Consejo Universitario. Politicas de la Universidad de Costa Rica para el año 2009. Universidad de Costa Rica, 2008. 
D`Antoni, M. Informe final. Taller de Necesidades Educativas Especiales. Departamento de Docencia Universitaria-Universidad de Costa Rica, 2008.

D`Antoni, M. y Vargas, M. C. "Capítulo 2. Taller de Necesidades Educativas Especiales: atención a estudiantes con necesidades educativas en la educación superior". Nuevos formatos para la función docente universitaria. San José, Costa Rica: Departamento de Docencia Universitaria-Escuela de Formación Docente-Universidad de Costa Rica, 2011.

Departamento de Docencia Universitaria, Escuela de Formación Docente. Programa Curso de Docencia Inclusiva: Desafíos y Acciones en la Educación Universitaria. Departamento de Docencia UniversitariaUniversidad de Costa Rica, 2011.

Maldonado, M. "El trabajo colaborativo en el aula universitaria". Laurus Revista de educación 13 (23). 2007: 263 278. En: <http://redalyc.uaemex.mx/ pdf/761/76102314>

Montoya, S. Propuesta de taller: entre (dis) capacidades e integraciones. San José, Costa Rica: Departamento de Docencia Universitaria-Universidad de Costa Rica, 2007.

Organización de las Naciones Unidas (onu). Convención sobre los derechos de las personas con discapacidad. Protocolo facultativo. México: onv, 2007.

Sánchez, J. A. El desarrollo profesional del docente universitario. 2002. En: $<w w w . u d u a l . o r g / c i d u / R e v i s t a / 22 /$ DesarrolloProfesional.htm>

Fecha de ingreso: 30/10/2012 Fecha de aprobación: 19/03/2013 
